\title{
Behavioral effects of Bj-PRO-7a, a proline-rich oligopeptide from Bothrops jararaca venom
}

\author{
L.C. Turones ${ }^{1}{ }^{1}$, K.R. da Cruz (ii) ${ }^{1}$, G. Camargo-Silva (ii) ${ }^{1}$, L.L. Reis-Silva (ii) ${ }^{1}$, D. Graziani (i) ${ }^{1}$ \\ P.M. Ferreira ${ }^{1}$, P.M. Galdino (ii) ${ }^{2}$, G.R. Pedrino (ii) ${ }^{1}$, R. Santos (ii) ${ }^{3}$, E.A. Costa ${ }^{2}{ }^{2}$, \\ D. lanzer (ii) ${ }^{1 *}$, and C.H. Xavier (ii) ${ }^{1 *}$
}

${ }^{1}$ Laboratório de Neurobiologia de Sistemas, Departamento de Ciências Fisiológicas, Instituto de Ciências Biológicas, Universidade Federal de Goiás, Goiânia, GO, Brasil

${ }^{2}$ Laboratório de Farmacologia de Produtos Naturais e Sintéticos, Departamento de Farmacologia, Instituto de Ciências Biológicas, Universidade Federal de Goiás, Goiânia, GO, Brasil ${ }^{3}$ Departamento de Fisiologia e Biofísica, Universidade Federal de Minas Gerais, Belo Horizonte, MG, Brasil

\begin{abstract}
The heptapeptide Bj-PRO-7a, isolated and identified from Bothrops jararaca $(B j)$ venom, produces antihypertensive and other cardiovascular effects that are independent on angiotensin converting enzyme inhibition, possibly relying on cholinergic muscarinic receptors subtype $1\left(\mathrm{M}_{1} \mathrm{R}\right)$. However, whether Bj-PRO-7a acts upon the central nervous system and modifies behavior is yet to be determined. Therefore, the aims of this study were: i) to assess the effects of acute administration of $B j-$ PRO-7a upon behavior; ii) to reveal mechanisms involved in the effects of Bj-PRO-7a upon locomotion/exploration, anxiety, and depression-like behaviors. For this purpose, adult male Wistar (WT, wild type) and spontaneous hypertensive rats (SHR) received intraperitoneal injections of vehicle $(0.9 \% \mathrm{NaCl})$, diazepam $(2 \mathrm{mg} / \mathrm{kg})$, imipramine $(15 \mathrm{mg} / \mathrm{kg}), B j-\mathrm{PRO}-7 \mathrm{a}(71,213 \mathrm{or}$ $426 \mathrm{nmol} / \mathrm{kg}$ ), pirenzepine (852 nmol $/ \mathrm{kg}), \alpha$-methyl-DL-tyrosine $(200 \mathrm{mg} / \mathrm{kg})$, or chlorpromazine $(2 \mathrm{mg} / \mathrm{kg})$, and underwent elevated plus maze, open field, and forced swimming tests. The heptapeptide promoted anxiolytic and antidepressant-like effects and increased locomotion/exploration. These effects of $B j-P R O-7 a$ seem to be dependent on $M_{1} R$ activation and dopaminergic receptors and rely on catecholaminergic pathways.
\end{abstract}

Key words: Bj-PRO-7a; Snake venom; Neuroactive compounds; Anxiety; Depression; Behavior

\section{Introduction}

Anxiety and depression are considered the principal debilitating diseases in the world, capable of compromising human wellness. Both diseases are highly prevalent and, as a consequence, there are substantially negative social and economic impacts. Because of the global incidence, developing new and efficient drugs is worth the effort, as the therapeutic arsenal currently available either displays delayed effects or shows no response in many cases of anxiety and depression (1). Therefore, venoms may represent a rich source of promising molecules, which includes neuroactive compounds and polypeptides (2).

Seminal research with the venom of Bothrops jararaca $(B j)$, a pit viper broadly distributed in South America, resulted in breakthrough discoveries of two compounds: bradykinin potentiating factors (BPFs) and bradykinin (Bk) itself. Subsequently, these BPFs were isolated and identified as peptides, formerly named bradykinin potentiating peptides (BPPs). Nevertheless, some BPPs were not capable of potentiating the Bk effects in pharmacological studies and were thus renamed as proline-rich oligopeptides (PROs) according to the similarity in their primary structure. The Bj-PROs have 5-17 amino acids residues with proline residues at $\mathrm{C}$-terminal and pyroglutamic acid at $\mathrm{N}$-terminal. The latter is a feature that confers high resistance against proteolytic activity exerted by some enzymes [for review, see Camargo et al. (3)].

As reported in the review by Camargo et al. (3), the first peptides identified by the scientists Sergio Henrique Ferreira and colleagues (1970) were capable of inhibiting the catalytic site of angiotensin converting enzyme (ACE), as shown by in vitro assays. Following the advances that uncovered the importance of the renin-angiotensin system to the cardiovascular homeostasis, several Bj-PROs analogues were synthesized and tested, presenting

Correspondence: C.H. Xavier: <carlosxavier@ufg.br>

${ }^{*}$ These authors contributed equally to his work. 
therapeutic potential to treat hypertension and other cardiovascular diseases. One of these molecules, captopril, presented major oral activity and was the first ACE inhibitor (ACEi) commercially available. However, this ACEi presented side effects, which encouraged further research. The study of the two ACE domains by Wei and coworkers and the discovery of Bj-PROs new sequences allowed for additional progress (3). Newly described peptides such as $B j$-PROs 7a, 11e, and 12b do not present affinity to ACE C-terminal domain, which is responsible for converting angiotensin I to angiotensin II. Intriguingly, these peptides evoked antihypertensive effects that were concomitant to bradycardia, through mechanisms that go far beyond those involving ACE inhibition (4).

$B j-P R O-7 a$, identified and isolated in the $B j$ crude venom, is composed by seven amino acids residues with an aspartic residue in $\mathrm{N}$-terminal position (Supplementary Figure S1) (5). The heptapeptide presented a potent and long-lasting anti-hypertensive effect in spontaneous hypertensive rats (SHR) that did not involve ACE (6). In addition, the heptapeptide was found intact in mice urine $24 \mathrm{~h}$ after intraperitoneal (ip) injection, which suggests a high resistance to enzymatic degradation (7). Another study demonstrated that Bj-PRO-7a modulates calcium transients in neurons and activates cholinergic muscarinic receptors subtype $1\left(\mathrm{M}_{1} \mathrm{R}\right)$ in vitro (8). Therefore, $B j-\mathrm{PRO}$ $7 \mathrm{a}$ is able to act upon multiple targets and possibly affect centrally organized functions. In this regard, the hypothesis we raised is that Bj-PRO-7a would modify the behavior also by cholinergic mechanisms. Additionally, we tested whether $B j-P R O-7 a$ effects would rely on catecholaminergic paths, since catecholamines also play a role in the etiology of depression and anxiety and are frequently chosen as targets for pharmacological treatments (9). To assess whether the possible central effects evoked by the heptapeptide would be independent of cardiovascular effects, hypertensive and normotensive rats were used. The aim of this study was to assess Bj-PRO-7a effects on locomotion, exploration, anxiety, and depression-like behaviors, and the possible mechanisms involved.

\section{Material and Methods}

\section{General procedures}

Animals. Male normotensive Wistar rats (WT) and SHR (weighing 300-380 g) bred at the Central Animal Facility of the Federal University of Goiás (UFG, Brazil) were randomly allocated to polypropylene cages $(47 \times 31 \times 16 \mathrm{~cm})$, five rats per cage, with water and food ad libitum, under controlled temperature and light/ dark cycle of $12 / 12 \mathrm{~h}$. Systolic blood pressure (SBP) was measured by tail plethysmography in SHRs to confirm hypertension (SHR: $194 \pm 7$ vs WT: $136 \pm 6 \mathrm{mmHg}$; $P<0.05)$. When the animals reached 15 weeks of age, SBP was recorded for 4 days. The rats were pre-warmed to approximately $38^{\circ} \mathrm{C}$ for $15 \mathrm{~min}$ to dilate the tail artery.
An inflatable cuff and pressure receptor were placed on the tail; both were coupled to a transducer and connected to an acquisition data system (PowerLab 4/25, ML0380/D, ADInstruments, Australia). The arterial pulse was measured by the inflatable cuff.

All experiments were approved by our local institutional animal welfare committee (CEUA/UFG protocol number 018/2015) and were performed in accordance with the Brazilian Federal Law No. 11.794. We made all efforts to minimize the number of animals used.

Drugs and reagents. All drugs were diluted in sterile saline $(0.9 \% \mathrm{NaCl})$, which was the vehicle of choice. The drugs tested were: i) Bj-PRO-7a (GenOne Biotechnology, Brazil) at doses of 71,213 , or $426 \mathrm{nmol} / \mathrm{kg}$ already tested in a previous study (6); ii) the specific antagonist of $M_{1} R$ pirenzepine (Sigma, USA) at the dose of $852 \mathrm{nmol} / \mathrm{kg}$; iii) the benzodiazepine diazepam (Sigma) at the dose of $2 \mathrm{mg} / \mathrm{kg}$, used as positive control for anxiety and locomotion tests; iv) the tricyclic antidepressant imipramine (Sigma) at the dose of $15 \mathrm{mg} / \mathrm{kg}$, used as positive control in depressive tests; $v$ ) the inhibitor of tyrosine hydroxylase $\alpha$-methyl-DL-tyrosine (AMPT) (Sigma) at the dose of $200 \mathrm{mg} / \mathrm{kg}$, used to reveal the catecholamines contribution; vi) the non-specific dopaminergic antagonist chlorpromazine (Sigma) at the dose of $2 \mathrm{mg} / \mathrm{kg}$, used to assess the possible contribution of dopamine receptors. All drugs and vehicle were injected ip.

\section{Behavioral tests}

On the experimental day, the rats were transported from the sectorial animal facility to the laboratory where experiments were performed and allocated in a separate room during $1 \mathrm{~h}$ for adaptation. Then, rats received vehicle or drugs according to the experiments - these administrations were conducted by the same person to avoid handling stress. All experiments were performed in a separate, quiet, and dimly illuminated room to prevent animals' communication and possible interferences during the experimental trials.

Elevated plus maze (EPM). Anxiety was evaluated with the EPM test (10). The maze consisted of two open arms $(50 \times 10 \times 1 \mathrm{~cm})$ and two closed arms $(50 \times 10 \times$ $40 \mathrm{~cm})$ connected by a central platform $(10 \times 10 \mathrm{~cm})$. The maze was elevated $50 \mathrm{~cm}$ from the ground and a video camera was fixed above the maze to record the rats' movements for posterior analysis. The maze was cleaned with $10 \%$ alcohol between experiments to prevent olfactory clues. At the start of the trial, each rat was placed in the center of the maze facing one of the closed arms. Animals were allowed to explore the maze for $5 \mathrm{~min}$. Six variables were measured: 1) time spent in the open arms; 2) number of entries into the open arms; 3 ) time spent in the closed arms (the placement of all four paws in the arms); 4) number of entries into the closed arms; 5 ) time spent in the central platform; and 6) number of total entries. A higher time and frequency in open arms, compared to vehicle, indicated an 
anxiolytic effect. In addition, the number of entries into the closed arms and the total entries also indicated general activity or locomotor activity, which was confirmed in the open field test.

Open field test (OF). Locomotion and exploration were evaluated in the OF test (10). The field was a round arena (120 cm in diameter) surrounded by a wall of $40 \mathrm{~cm}$, with the surface subdivided into 12 squares. A video camera was fixed above the field to record the rats' movements for posterior analysis. The OF test was performed immediately after the EPM test. The field was cleaned with $10 \%$ alcohol between experiments to prevent olfactory clues. Each rat was placed in the center of the field and was allowed to explore the apparatus for $5 \mathrm{~min}$. Six variables were measured: 1) time spent in the periphery; 2) time spent in the center; 3) immobility time; 4) number of square crossings; 5) number of rearing episodes; and 6) number of self-grooming (including paw licking, nose/face grooming, and head washing). Immobility, crossings, and selfgrooming were the locomotor outcomes; rearing episodes were recorded as exploratory behavior.

Forced swimming (FS). The FS test was performed as previously described (10). On the first day, rats were placed in a cylinder of polyvinyl chloride $(24 \mathrm{~cm}$ of diameter and $60 \mathrm{~cm}$ of high) filled by a water column $\left(42 \mathrm{~cm}\right.$ at $25 \pm 1^{\circ} \mathrm{C}$ ) and were allowed to swim for $15 \mathrm{~min}$. Then, the rats were dried and placed in a warmed cage at approximately $38^{\circ} \mathrm{C}$. Subsequently, the first drug administration ( $24 \mathrm{~h}$ before FS test) was performed. On the next day, the second and third drug administrations were performed, 5 and $1 \mathrm{~h}$ before the FS test, respectively. During the test, each rat was allowed to swim for $6 \mathrm{~min}$. The experiment was recorded by a video camera fixed above the cylinder and the last 4 min were used for posterior analysis. The cylinder was cleaned with $10 \%$ alcohol and the water was removed after each experiment to prevent olfactory clues. The immobility time (when the rat stopped the struggling movements and floated on the water, making only movements to keep the head above the water surface) was a measure of depressive behavior.

\section{Experimental design}

Experiment 1: Effects of Bj-PRO-7a on anxiety and locomotion/exploration. WT and SHR received an ip injection of vehicle, diazepam, or Bj-PRO-7a $(71,213$, and $426 \mathrm{nmol} / \mathrm{kg}$ ) $30 \mathrm{~min}$ before the EPM test. After the end of EPM testing, rats were tested in the OF.

Experiment 2: Involvement of central $M_{1} R$ in the responses evoked by Bj-PRO-7a on anxiety and locomotion/exploration. We used a systemic (ip) injection of pirenzepine to maintain the same route of administration for all drugs, which also ensured a minimally invasive method. Although pirenzepine presents low liposolubility, previous studies demonstrated that it crosses the blood brain barrier, reaches the central nervous system (11), and produces central effects $(12,13)$.

WT rats received pirenzepine (852 nmol/kg, ip) $30 \mathrm{~min}$ before injection of vehicle or Bj-PRO-7a (426 nmol/kg, ip). Thirty min after the last injection (vehicle or Bj-PRO-7a, ip), the EPM test was performed. Following the end of EPM, rats were tested in the OF.

Experiment 3: Involvement of catecholaminergic pathways in the effects evoked by Bj-PRO-7a on anxiety and exploration/locomotion. WT rats received AMPT $(200 \mathrm{mg} / \mathrm{kg}$, ip) $1 \mathrm{~h}$ before an injection of vehicle or Bj-PRO-7a (426nmol/kg, ip). Thirty min after vehicle or Bj-PRO-7a administration the EPM test was performed. Following the end of EPM, rats were tested in the OF.

Experiment 4: Contribution of dopaminergic receptors to the effects evoked by Bj-PRO-7a on anxiety and exploration/locomotion. WT rats received chlorpromazine $(2 \mathrm{mg} / \mathrm{kg}$, ip) $30 \mathrm{~min}$ before vehicle or Bj-PRO-7a (426 nmol/kg, ip). Thirty min after vehicle or Bj-PRO-7a administration the EPM test was performed followed by the OF.

Experiment 5: Effects of Bj-PRO-7a on depression-like behavior. WT rats and SHRs received three injections of vehicle, imipramine (15 mg/kg, ip), or Bj-PRO-7a (71, 213 or $426 \mathrm{nmol} / \mathrm{kg}$, ip) 24,5 , and $1 \mathrm{~h}$ before the FS test.

Experiment 6: Involvement of $M_{1} R$ in the responses evoked by Bj-PRO-7a on depression-like behavior. WT rats received three injections of vehicle or Bj-PRO-7a (426 $\mathrm{nmol} / \mathrm{kg}$, ip) 24,5 , and $1 \mathrm{~h}$ before the FS test. Pirenzepine (852 nmol/kg, ip) was given 30 min before the last vehicle or Bj-PRO-7a administration.

Experiment 7: Involvement of catecholaminergic pathways in the responses evoked by Bj-PRO-7a on depression-like behavior. WT rats received three injections of vehicle or Bj-PRO-7a (426 nmol/kg, ip) at 24, 5, and $1 \mathrm{~h}$ before the FS test. $\alpha$-methyl-DL-tyrosine $(200 \mathrm{mg} / \mathrm{kg}$, ip) was injected $1 \mathrm{~h}$ before the last vehicle or Bj-PRO-7a administration.

Experiment 8: Contribution of dopaminergic receptors to the responses evoked by Bj-PRO-7a on depression-like behavior. WT rats received three injections of vehicle or Bj-PRO-7a (426 nmol/kg, ip) at 24, 5, and $1 \mathrm{~h}$ before the FS test. Chlorpromazine ( $2 \mathrm{mg} / \mathrm{kg}$, ip) was injected $30 \mathrm{~min}$ before the last vehicle or Bj-PRO-7a administration.

\section{Statistical analysis}

The data are reported as means \pm SE and were analyzed by two-way ANOVA followed by Bonferroni's post hoc test (experiments 1 and 5, factors strain and treatment) or one-way ANOVA followed by Fisher's post hoc test (experiments 2-4 and 6-8). Standard deviation was set as the exclusion parameter for statistical analysis. All data were analyzed using GraphPad Prism 6.0 (GraphPad Software, Inc., USA). The level of significance was fixed at $\mathrm{P}<0.05$. 


\section{Results}

\section{Bj-PRO-7a produced an anxiolytic-like effect and locomotor/exploratory responses}

As expected, the treatment with diazepam increased the time and the number of entries in open arms in WT rats (Figure $1 \mathrm{~A}$ and $\mathrm{D}$ ). The same anxiolytic effect was observed in SHRs. Bj-PRO-7a decreased anxiety in WT rats, as revealed by the increases in the time and number of entries into open arms of the maze (Figure $1 A$ and $D$ ). Similar effects were observed in the time spent in open arms by SHRs injected with the heptapeptide and in the number of entries into open arms, except at the dose of $71 \mathrm{nmol} / \mathrm{kg}$. Bj-PRO-7a also reduced the time spent in closed arms, except for the dose of $71 \mathrm{nmol} / \mathrm{kg}$ (Figure 1B). The treatment with Bj-PRO-7a did not change the number of entries into the closed arms (Figure 1E). Interestingly, the total number of entries was increased by Bj-PRO-7a at doses of $426 \mathrm{nmol} / \mathrm{kg}$ in WT rats (Figure 1F). The time spent in the maze center did not present significant changes with $B j-P R O-7 a$ treatment.

The treatment with $B j-P R O-7 a$ reduced the time spent in the periphery (Figure 2A) and increased the time spent in the center (Figure 2B) in SHRs, but not in WT rats. Additionally, the treatment with Bj-PRO-7a at $426 \mathrm{nmol} / \mathrm{kg}$ increased locomotion and exploration, as revealed by reductions in the immobility time (Figure $2 \mathrm{C}$ ), increases in the number of crossings (Figure 2D), and rearing episodes (Figure 2E). The heptapeptide did not significantly modify the self-grooming (Figure $2 \mathrm{~F}$ ).

Since the results obtained from these first experiments showed small differences in the effects of Bj-PRO-7a between strains, the subsequent trials were conducted only in WT rats. Considering that $426 \mathrm{nmol} / \mathrm{kg} \mathrm{Bj-PRO-7a}$ was the dose that produced pronounced effects, this dose was chosen to investigate the subsequent mechanistic hypothesis using pirenzepine (PZP), $\alpha$-methyl-DL-tyrosine (AMPT), and chlorpromazine (CLP).
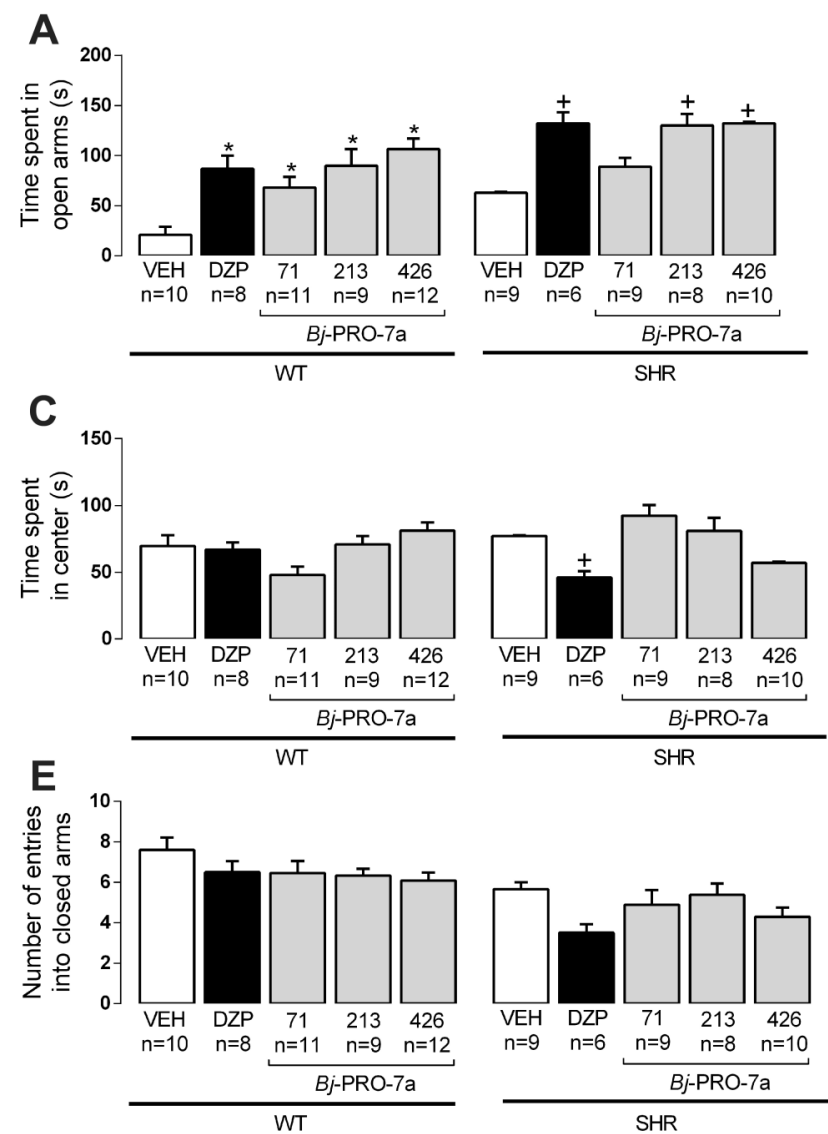
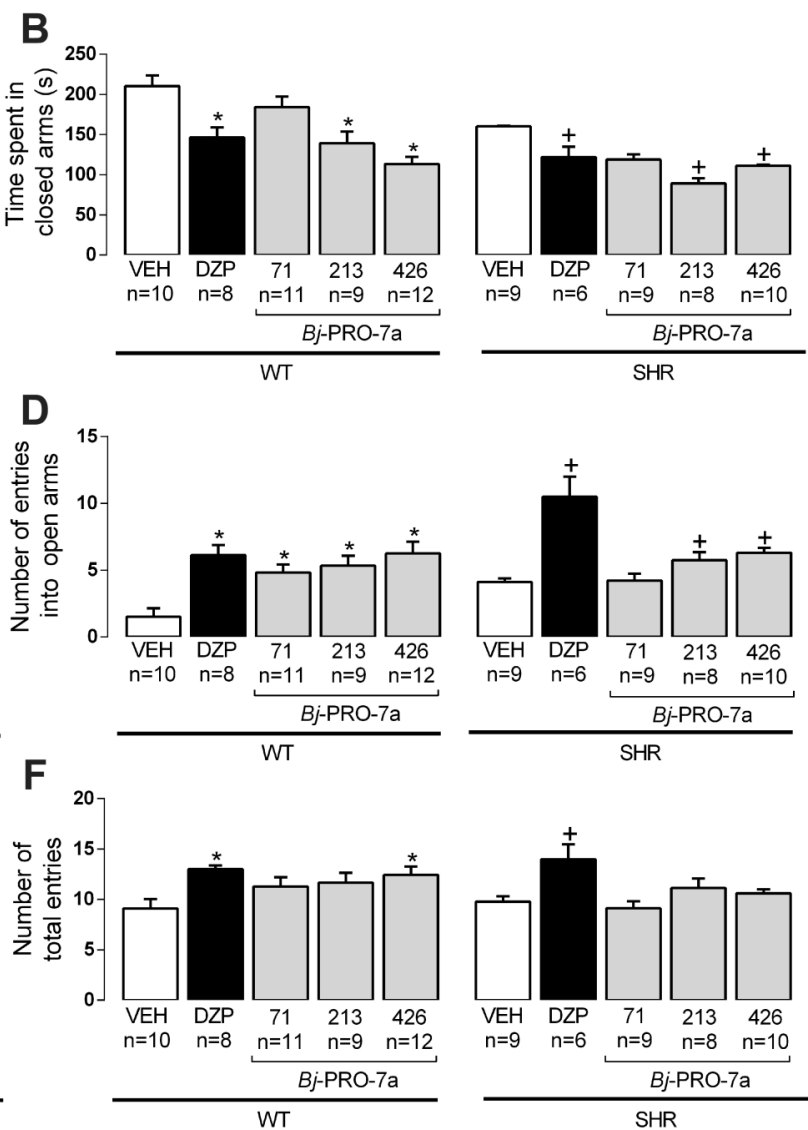

Figure 1. Effects of Bj-PRO-7a on anxiety-like behaviors in the elevated plus maze in Wistar (WT) and spontaneously hypertensive rats (SHRs). A, Time spent in open arms; B, Time spent in closed arms; C, Time spent in center; D, Number of entries into open arms; E, Number of entries into closed arms; $\mathbf{F}$, Number of total entries. Experimental groups: VEH: vehicle (0.9\% NaCl); DZP: diazepam (2 mg/kg); Bj-PRO-7a $(71,213$, or $426 \mathrm{nmol} / \mathrm{kg})$. Data are reported as means $\pm \mathrm{SE} .{ }^{*} \mathrm{P}<0.05$ vs VEH WT; ${ }^{+} \mathrm{P}<0.05$ vs VEH SHR (two-way analysis of variance followed by Bonferroni's post hoc test). 
A

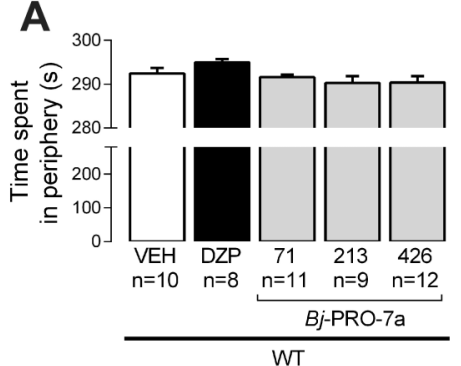

C

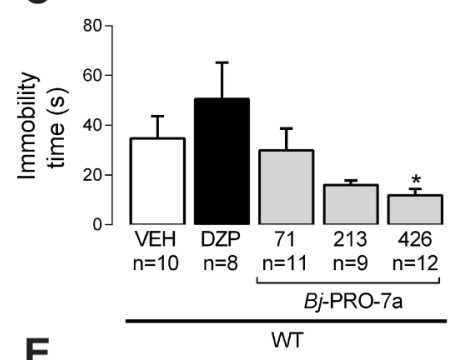

$E$

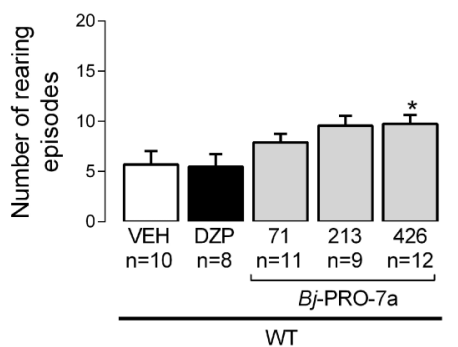

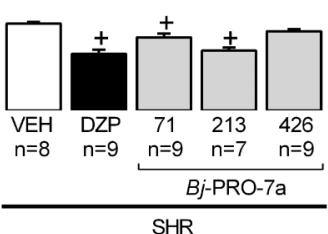
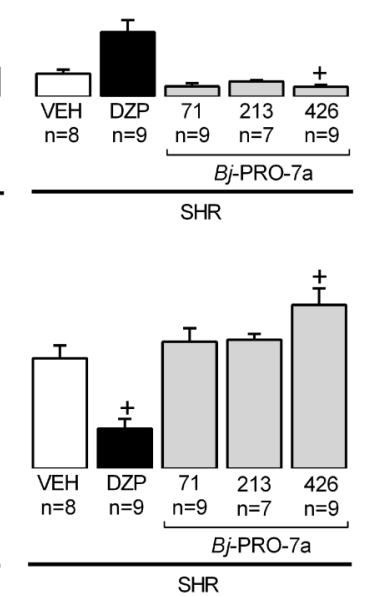

B
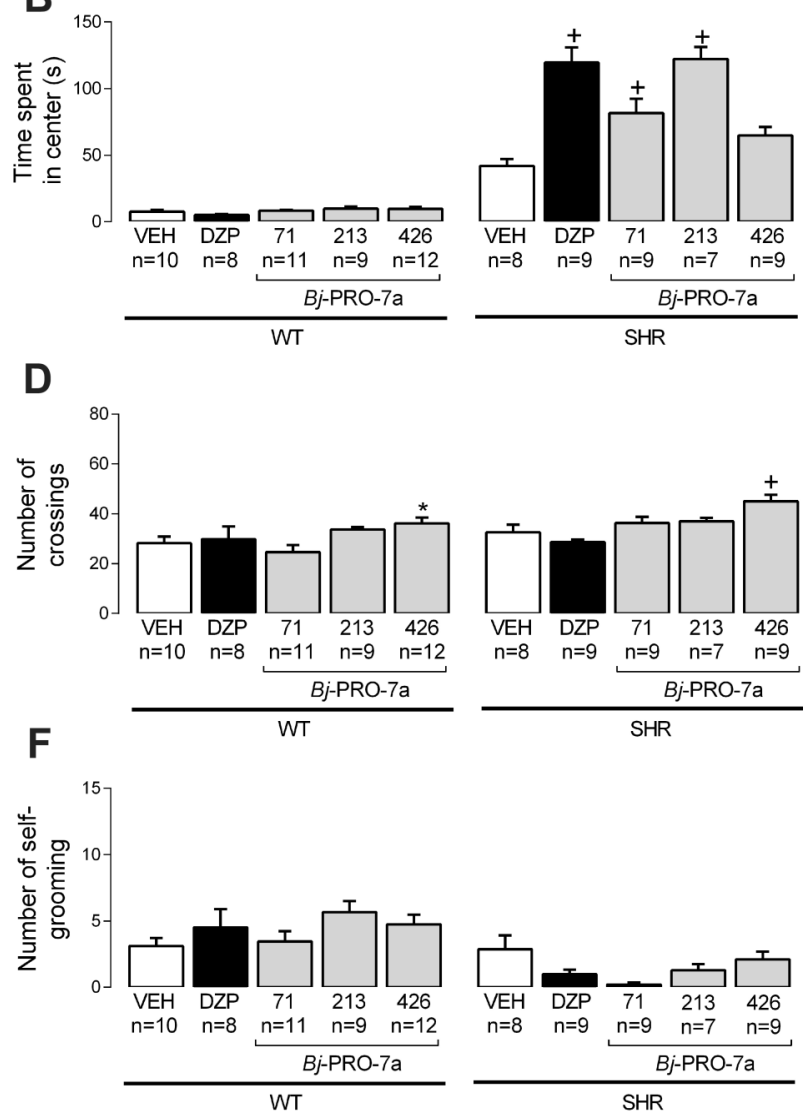

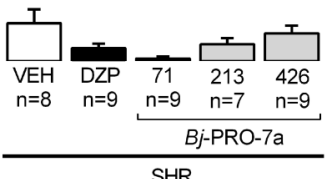

Figure 2. Effects of Bj-PRO-7a on locomotion and exploration activities in the open field in Wistar (WT) and spontaneously hypertensive rats (SHRs). A, Time spent in periphery; B, Time spent in center; C, Immobility time; D, Number of crossings; E, Number of rearing episodes; F, Number of self-grooming episodes. Experimental groups: VEH: vehicle $(0.9 \% \mathrm{NaCl})$; DZP: diazepam (2 mg/kg); Bj-PRO-7a $\left(71,213\right.$, or $426 \mathrm{nmol} / \mathrm{kg}$ ). Data are reported as means $\pm \mathrm{SE}$. ${ }^{*} \mathrm{P}<0.05 \mathrm{vs} \mathrm{VEH}$ WT; ${ }^{+} \mathrm{P}<0.05$ vs VEH SHR (two-way analysis of variance followed by Bonferroni's post hoc test).

\section{Activation of muscarinic receptor subtype 1 was important to the anxiolysis promoted by Bj-PRO-7a}

PZP $(852 \mathrm{nmol} / \mathrm{kg})$ did not promote significant alterations in EPM and OF tests compared to the vehicle group (Figures 3 and 4). Interestingly, the combined injection of $B j-P R O-7 a$ with PZP reverted the increases in the time and number of entries into the open arms of EPM evoked by Bj-PRO-7a (Figure 3A and D). In addition, PZP reverted the reduction in the time spent in closed arms produced by the heptapeptide (Figure 3B). This demonstrated that the anxiolytic-like effect of $B j-P R O-7 a$ depends on $M_{1} R$. However, the effects of Bj-PRO-7a (reduction of immobility time, increase in crossing and rearing episodes) in the OF test were not modified by the antagonism of $M_{1} R$ (Figure $4 C-F$ ).

\section{The catecholaminergic system was involved in} anxiolytic-like effects evoked by Bj-PRO-7a

AMPT $(200 \mathrm{mg} / \mathrm{kg})$ reduced the locomotion in the EPM test compared to vehicle, as revealed by the decreases in number of entries into closed arms and number of total entries (Figure 5E and F). Additionally, catecholamine depletion promoted effects that were inverse to those evoked by Bj-PRO-7a, except in the number of entries into the closed arms of the maze (Figure 5F). Noticeably, the anxiolysis evoked by the heptapeptide was attenuated by simultaneous administration of $\alpha$-methyl-DL-tyrosine with Bj-PRO-7a (Figure 5A, B, and D).

In OF, AMPT reduced the locomotion (Figure 6C) and exploration (Figure 6E) compared to vehicle. The catecholaminergic depletor promoted effects that were inverse to those evoked by the heptapeptide in locomotion and exploration parameters (Figure $6 \mathrm{C}-\mathrm{F}$ ). Interestingly, the concomitant injection of AMPT and Bj-PRO-7a reverted the increases in locomotion and exploration evoked by the heptapeptide in the OF (Figure $6 \mathrm{C}-\mathrm{F}$ ). These results suggested that catecholaminergic pathways are important for Bj-PRO-7a to perform their effects on locomotion and exploration. 

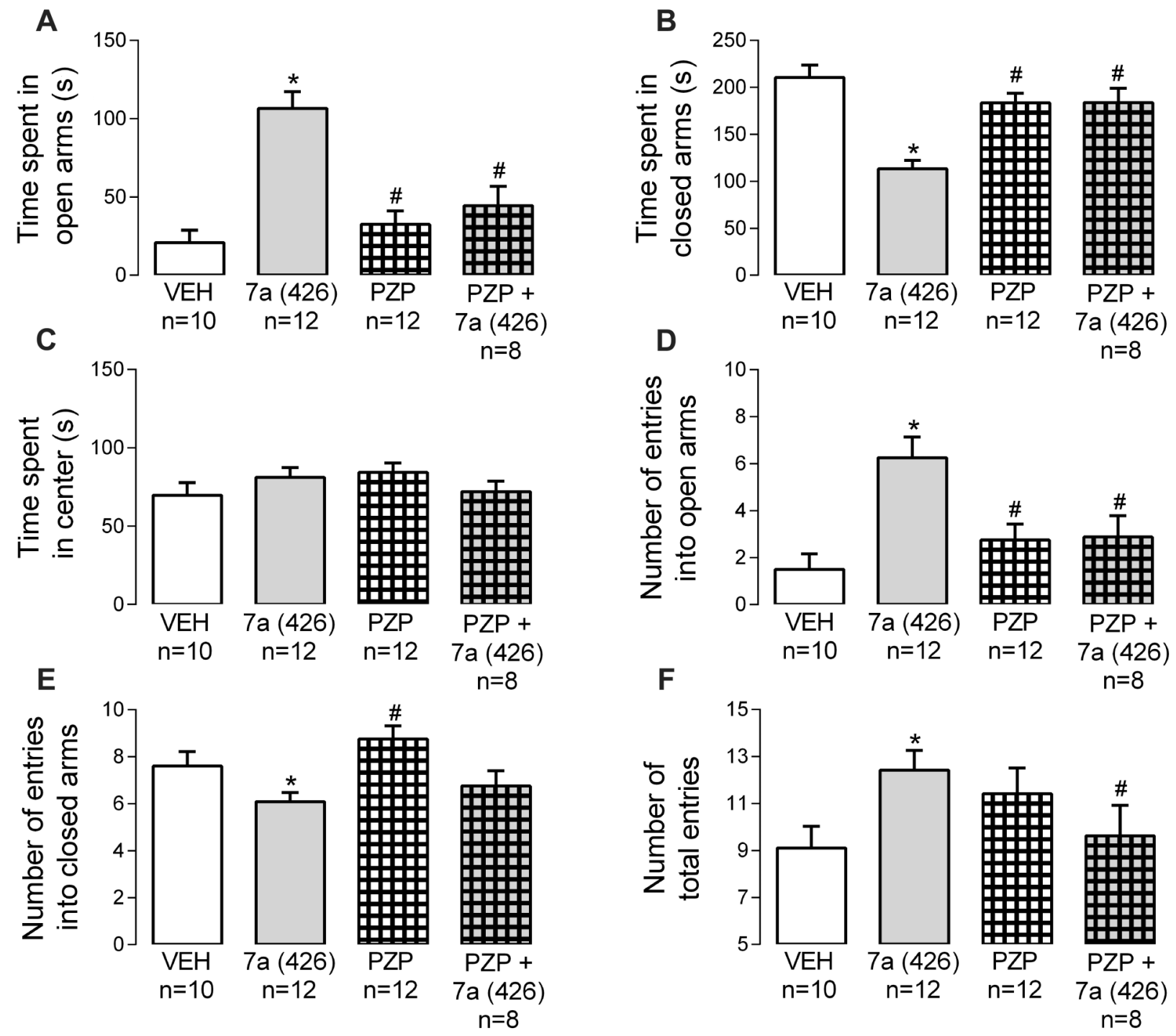

Figure 3. Contribution of muscarinic receptor subtype 1 (as revealed by the antagonism with pirenzepine) to the anxiety-like effect evoked by Bj-PRO-7a in the elevated plus maze. A, Time spent in open arms; B, Time spent in closed arms; C, Time spent in center; D, Number of entries into open arms; E, Number of entries into closed arms; $\mathbf{F}$, Number of total entries. Experimental groups: VEH: vehicle $(0.9 \% \mathrm{NaCl})$; 7a (426): Bj-PRO-7a (426 nmol/kg); PZP: pirenzepine (852 nmol/kg); PZP + 7a (426): co-administration of pirenzepine and Bj-PRO-7a (426 nmol/kg). Data are reported as means $\pm \mathrm{SE}$. ${ }^{*} \mathrm{P}<0.05$ vs vehicle; ${ }^{\#} \mathrm{P}<0.05$ vs $7 a(426)$ (one-way analysis of variance followed by Fisher's post hoc test).

\section{Dopaminergic receptors played a role in the} behavioral effects evoked by Bj-PRO-7a

CLP (2 mg/kg) promoted anxiolysis (Figure 7A, B, and D) compared to vehicle. This antagonism of dopaminergic receptors presented effects that were inverse to those evoked by Bj-PRO-7a in time spent in open and closed arms of the maze (Figure $7 \mathrm{~A}$ and $\mathrm{B}$ ). Intriguingly, the anxiolysis evoked by Bj-PRO-7a was reverted by its concomitant administration with CLP, as revealed by decreases in the time spent in the open arms and the number of entries into the maze area (Figure 7A and D). Therefore, the activity of dopaminergic receptors was required to promote the anxiolysis evoked $B j-P R O-7 a$.

In the OF, CLP provoked anxiolytic-like effects compared to vehicle (Figure $8 \mathrm{~A}$ and $\mathrm{B}$ ). Also, the antagonism of dopaminergic receptors reduced the locomotion and exploration activities compared to Bj-PRO-7a (Figure $8 \mathrm{C}-\mathrm{F}$ ). Intriguingly, the co-administration of CLP with Bj-PRO-7a reduced the immobility compared to vehicle. Conversely to what was found in the EPM, dopaminergic receptors did not 
A
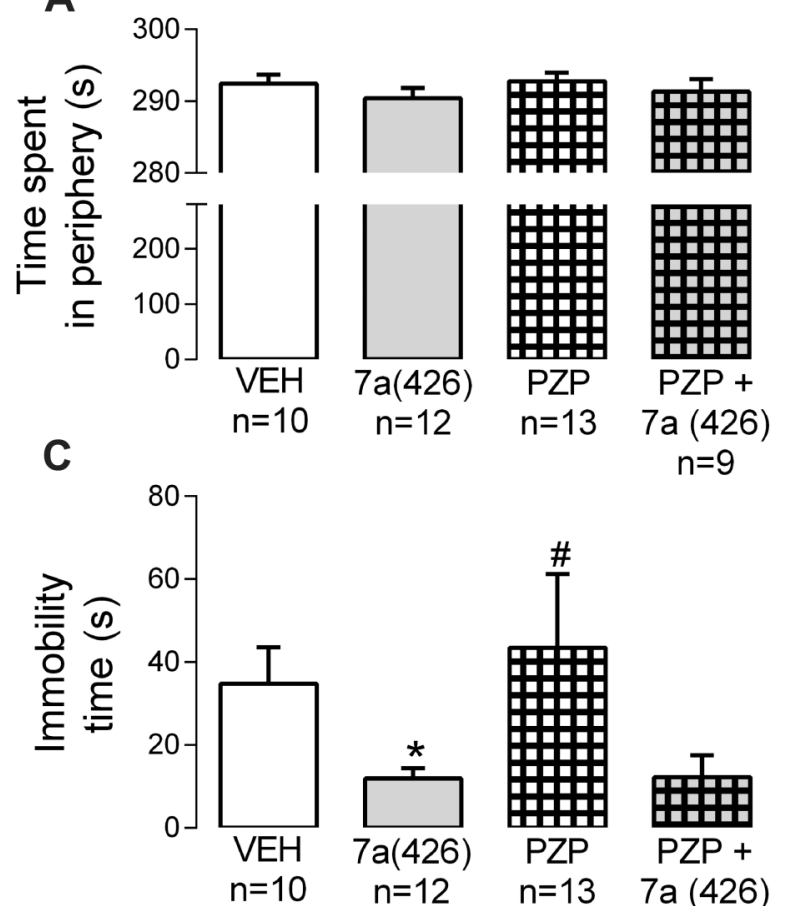

E

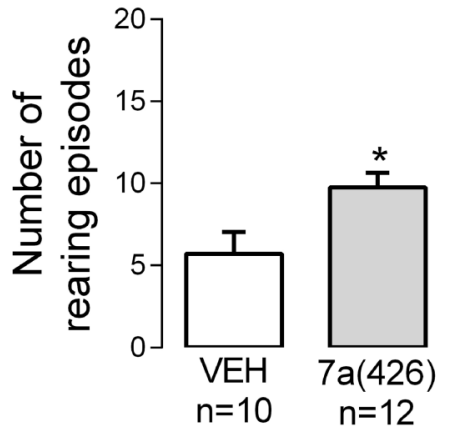

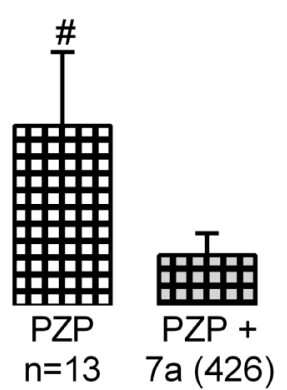

$\mathrm{n}=9$

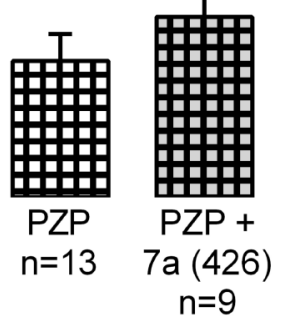

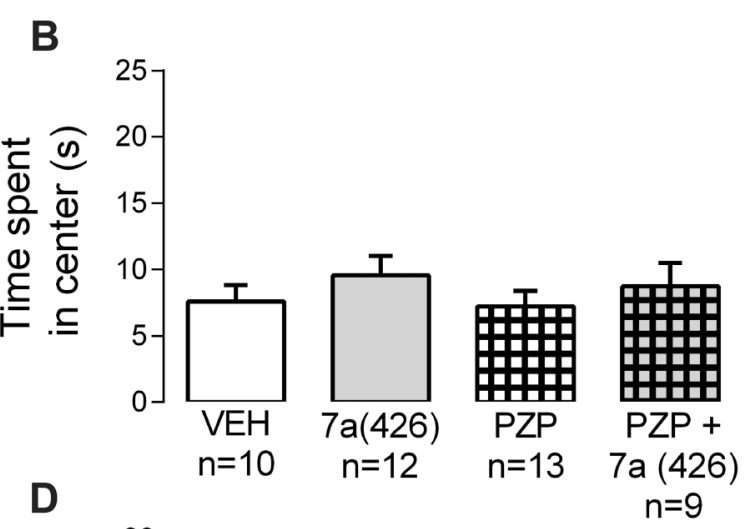

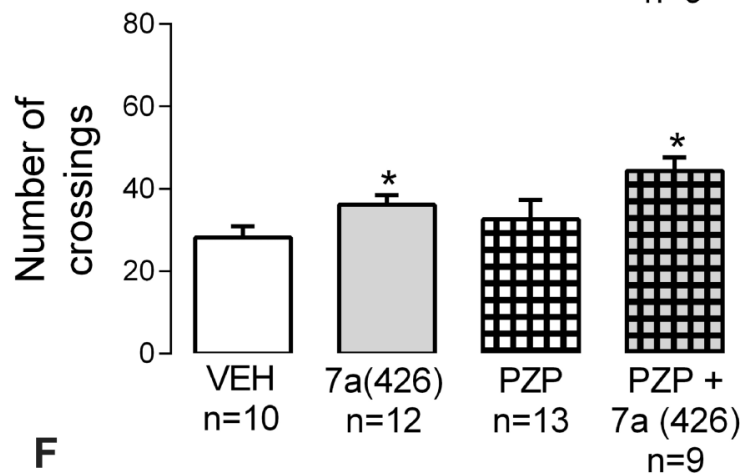

Figure 4. Contribution of muscarinic receptor subtype 1 (as revealed by the antagonism with pirenzepine) to the effects evoked Bj-PRO7a on locomotion/exploration in the open field. A, Time spent in periphery; B, Time spent in center; C, Immobility time; $\mathbf{D}$, Number of crossings; $\mathbf{E}$, Number of rearing episodes; F, Number of self-grooming episodes. Experimental groups: $\mathrm{VEH}$ : vehicle $(0.9 \% \mathrm{NaCl}) ; 7 \mathrm{a}$ (426): Bj-PRO-7a (426 nmol/kg); PZP: pirenzepine (852 nmol/kg); PZP + 7a (426): co-administration of PZP and Bj-PRO-7a (426 nmol/ $\mathrm{kg}$ ). Data are reported as means $\pm \mathrm{SE}$. ${ }^{*} \mathrm{P}<0.05$ vs vehicle; ${ }^{\sharp} \mathrm{P}<0.05$ vs $7 \mathrm{a}(426)$ (one-way analysis of variance followed by Fisher's post hoc test).

play a role in the locomotion and exploratory effects evoked by $B j-P R O-7 a$ (Figure $8 \mathrm{C}-\mathrm{F}$ ).

\section{Antidepressant-like actions of Bj-PRO-7a depended on catecholaminergic synthesis and on activation of $M_{1} R$}

As expected, the treatment with imipramine - a tricyclic antidepressant - reduced the immobility time compared to vehicle (Figure 9A). The treatment with
Bj-PRO-7a at doses of 71,213 , and $426 \mathrm{nmol} / \mathrm{kg}$ reduced the immobility time (Figure 9A) in normotensive and hypertensive rats during the FS test compared to vehicle. Considering that $B j-P R O-7 a$ produced the same antidepressant-like effect in both strains (normotensive and antihypertensive rats), the subsequent trials were conducted only in WT rats with vehicle and Bj-PRO-7a $(426 \mathrm{nmol} / \mathrm{kg})$ used to compare the effects of PZP, AMPT, and CLP. 


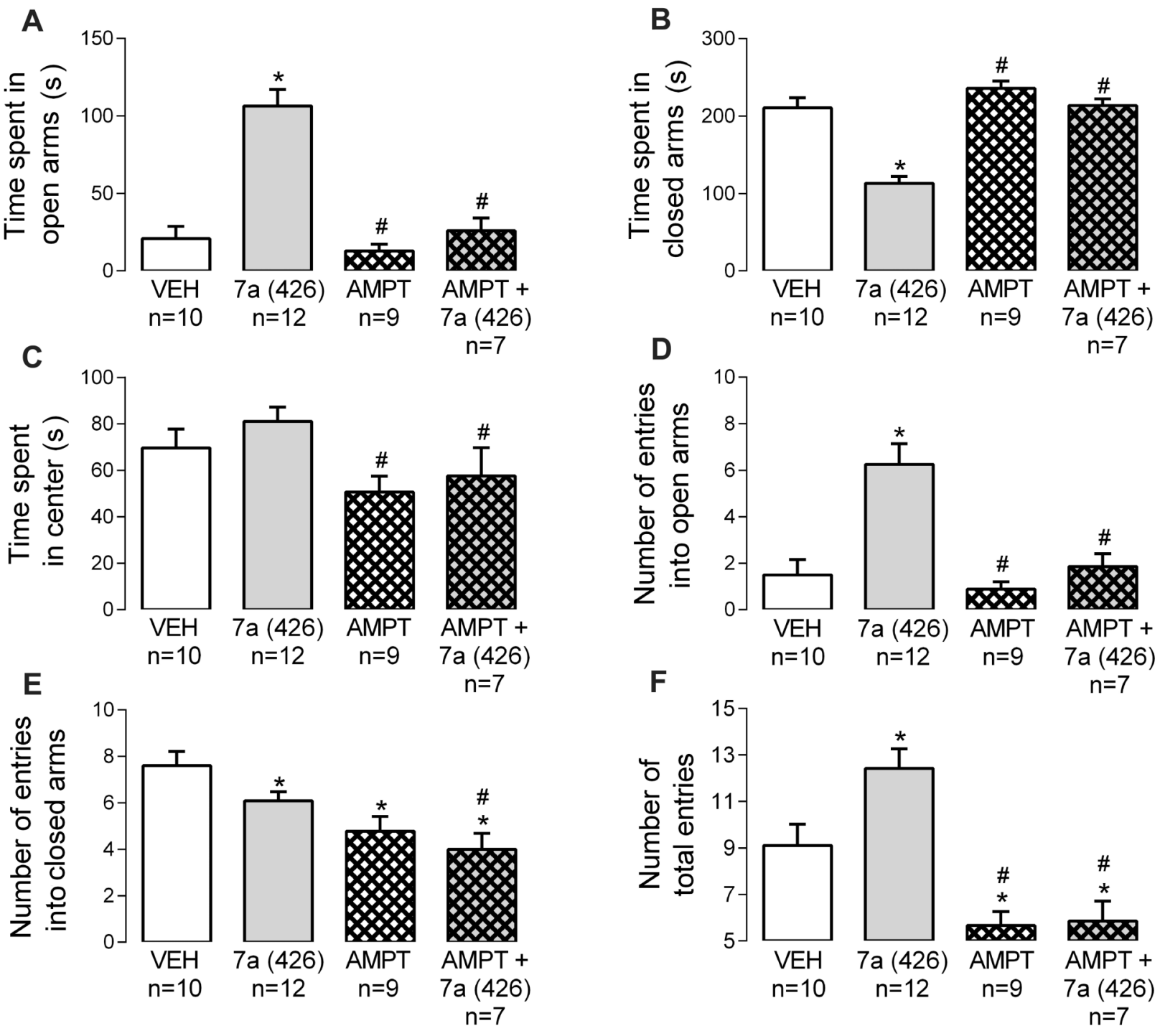

Figure 5. Contribution of catecholaminergic pathways to the anxiety-like effect evoked by Bj-PRO-7a in the elevated plus maze. A, Time spent in open arms; B, Time spent in closed arms; C, Time spent in center; D, Number of entries into open arms; $\mathbf{E}$, Number of entries into closed arms; F, Number of total entries. Experimental groups: VEH: vehicle (0.9\% NaCl); $7 \mathrm{a}$ (426): Bj-PRO-7a (426 nmol/kg); AMPT: $\alpha$-methyl-DL-tyrosine (200 mg/kg); AMPT + 7a (426): co-administration of $\alpha$-methyl-DL-tyrosine and Bj-PRO-7a (426 nmol $/ \mathrm{kg})$. Data are reported as means \pm SE. * $\mathrm{P}<0.05$ vs vehicle; ${ }^{\#} \mathrm{P}<0.05$ vs $7 \mathrm{a}(426)$ (one-way analysis of variance followed by Fisher's post hoc test).

The immobility time sampled following $M_{1} R$ antagonism with PZP did not differ from vehicle (Figure 9B). Interestingly, the co-administration of PZP with Bj-PRO-7a decreased the immobility time compared to vehicle (Figure 9B), but these values were still greater than those produced by Bj-PRO-7a. Therefore, the antidepressant-like effects promoted by the heptapeptide partially depend on $M_{1} R$.

Catecholaminergic depletion with AMPT increased the immobility time in the FS test (Figure 9C). Consequently, this catecholaminergic depletion was able to revert the antidepressant-like effect evoked by Bj-PRO-7a.
The antagonism of dopaminergic receptors with CLP did not modify the magnitude of the immobility time measured in the group injected vehicle (Figure 9D). However, compared to Bj-PRO-7a, CLP increased the immobility time. In this regard, the co-administration of CLP and Bj-PRO-7a decreased the amplitude of the antidepressant-like effect evoked by the heptapeptide, since the results obtained from this co-administration were comparable to vehicle (Figure 9D). Therefore, the antidepressant-like effects evoked by $B j-P R O-7 a$ relied on dopaminergic receptors. 
A

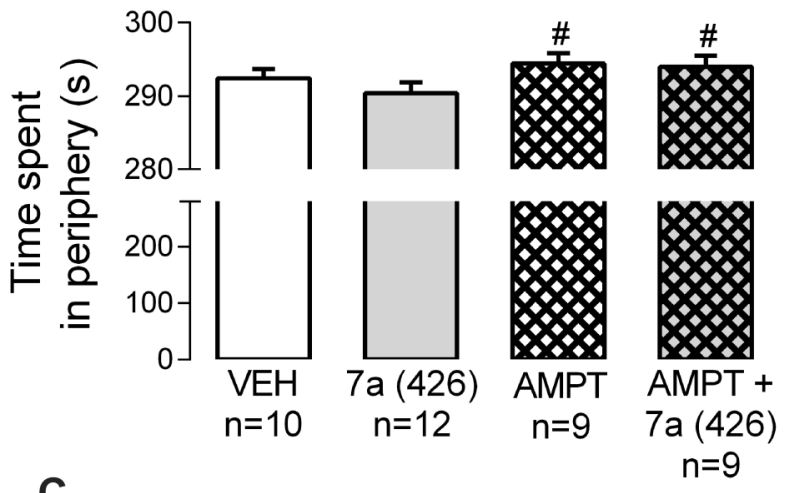

C

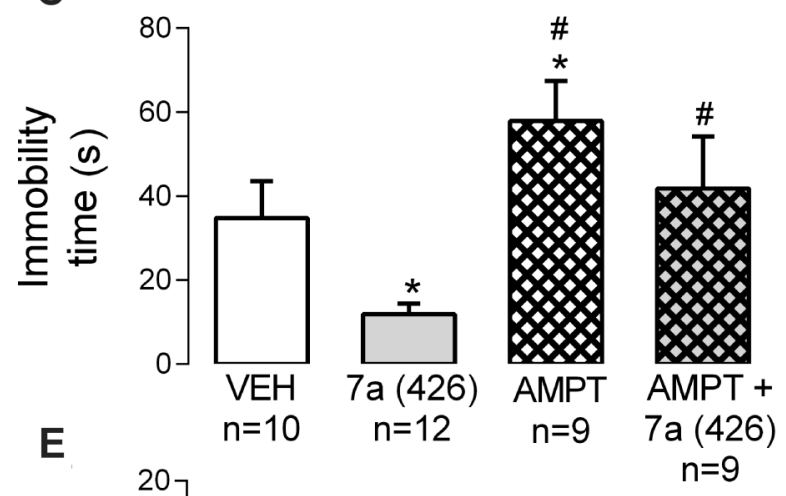

B

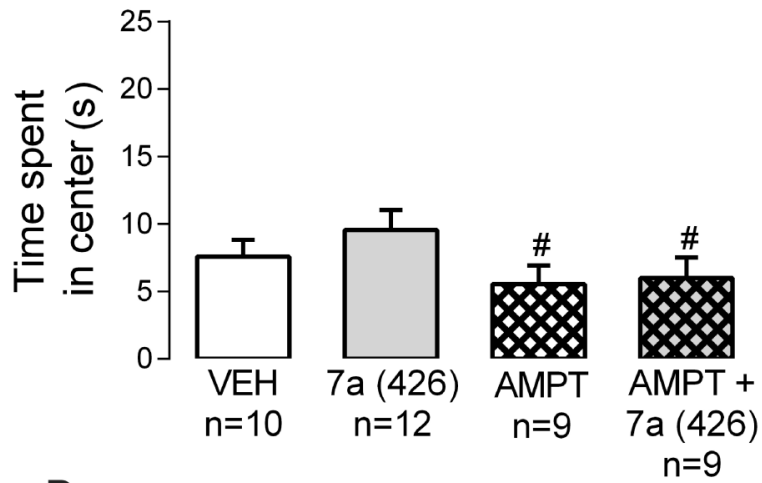

Figure 6. Contribution of catecholaminergic pathways (as revealed by the depleter $\alpha$-methyl-DL-tyrosine) to the effects evoked by BjPRO-7a on locomotion/exploration in the open field. A, Time spent in periphery; B, Time spent in center; C, Immobility time; D, Number of crossings; $\mathbf{E}$, Number of rearing episodes; $\mathbf{F}$, Number of self-grooming espisodes. Experimental groups: VEH: vehicle (0.9\% NaCl); 7 a (426): Bj-PRO-7a (426 nmol/kg); AMPT: $\alpha$-methyl-DL-tyrosine (200 mg/kg); AMPT + 7a (426): co-administration of $\alpha$-methyl-DL-tyrosine and Bj-PRO-7a (426 nmol/kg). Data are reported as means $\pm \mathrm{SE}$. ${ }^{*} \mathrm{P}<0.05$ vs vehicle; ${ }^{\#} \mathrm{P}<0.05$ vs $7 \mathrm{a}(426)$ (one-way analysis of variance followed by Fisher's post hoc test).

\section{Discussion}

The main findings of this study were: i) Bj-PRO-7a promoted anxiolytic and antidepressant-like effects in WT and SHRs; ii) the higher dose of the heptapeptide increased the locomotion and exploration of rats; iii) the antagonism of $M_{1} R$ by $P Z P$ reverted the anxiolytic-like effect and decreased the antidepressant-like effect evoked by $B j-P R O-7 a$; iv) catecholamines depletion, a result of inhibition of tyrosine hydroxylase activity by AMPT, reverted the anxiolytic-like and antidepressant-like effects caused by Bj-PRO-7a; and v) the antagonism of dopaminergic receptors by CLP reverted the anxiolytic-like effect and attenuated the antidepressant-like effect provoked by Bj-PRO-7a.

Snake venoms are cocktails of toxins composed by enzymatic and non-enzymatic bioactive polypeptides and in less proportion, carbohydrates, lipids, amino 


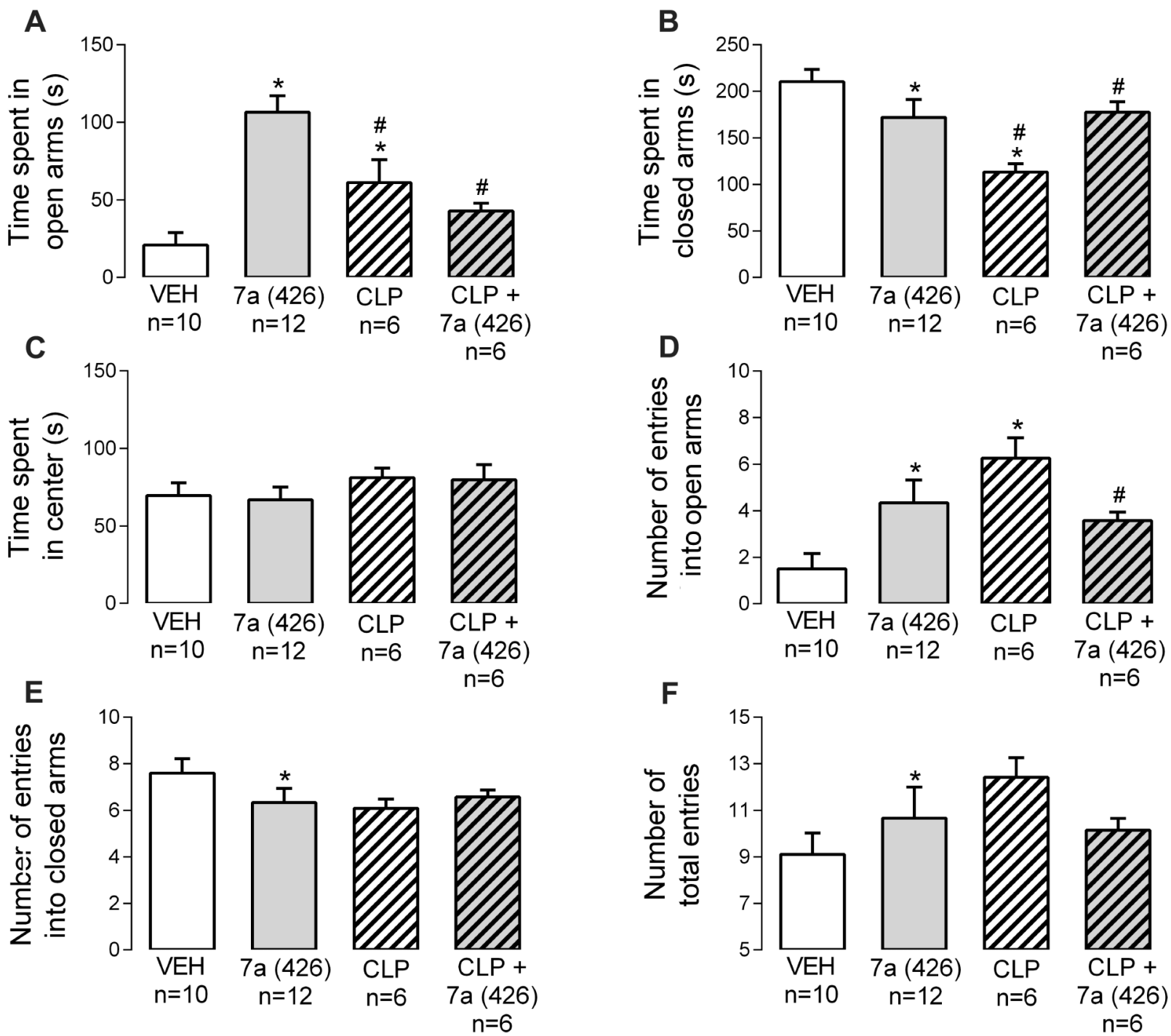

Figure 7. Contribution of dopaminergic receptors (as revealed by the antagonism with chlorpromazine) to the anxiety-like effect evoked by Bj-PRO-7a in the elevated plus maze. A, Time spent in open arms; $\mathbf{B}$, Time spent in closed arms; C, Time spent in center; $\mathbf{D}$, Number of entries into open arms; E, Number of entries into closed arms; $\mathbf{F}$, Number of total entries. Experimental groups: VEH: vehicle $(0.9 \%$ $\mathrm{NaCl}) ; 7 \mathrm{a}(426)$ : Bj-PRO-7a (426 nmol/kg); CLP: chlorpromazine (2 mg/kg); CLP + 7a (426): co-administration of CLP and Bj-PRO-7a $(426 \mathrm{nmol} / \mathrm{kg})$. Data are reported as mean $\pm \mathrm{SE}$. ${ }^{*} \mathrm{P}<0.05$ vs vehicle; ${ }^{\#} \mathrm{P}<0.05$ vs $7 \mathrm{a}$ (426) (one-way analysis of variance followed by Fisher's post hoc test).

acids, and minerals. The side effects triggered from envenoming by Bothrops jararaca bite are severe pain, acute renal failure, shock, intracranial hemorrhage, and other hemostatic disorders. The toxins displaying enzymatic activities, such as hydrolases, phosphodiesterases, and phospholipases, are known for converging actions towards prey immobilization and the subsequent intake and digestion. These enzymatic toxins may be either promiscuous or highly specific with regard to their molecular targets (2). However, the effects of these neuroactive compounds are not confined to the somatic nervous system, thus affecting behavior and other functions by crossing the blood-brain barrier and, as a consequence, these toxins are able to reach the central nervous system (CNS) (2). Furthermore, neuroactive compounds have a potent curarimimetic action on peripheral cholinergic transmission (14). Considering the multiplicity of venom components and the molecular targets, it is plausible to hypothesize that venoms previously known for being predominantly composed by hemotoxins would also be able to reach a degree of neurotoxicity. 

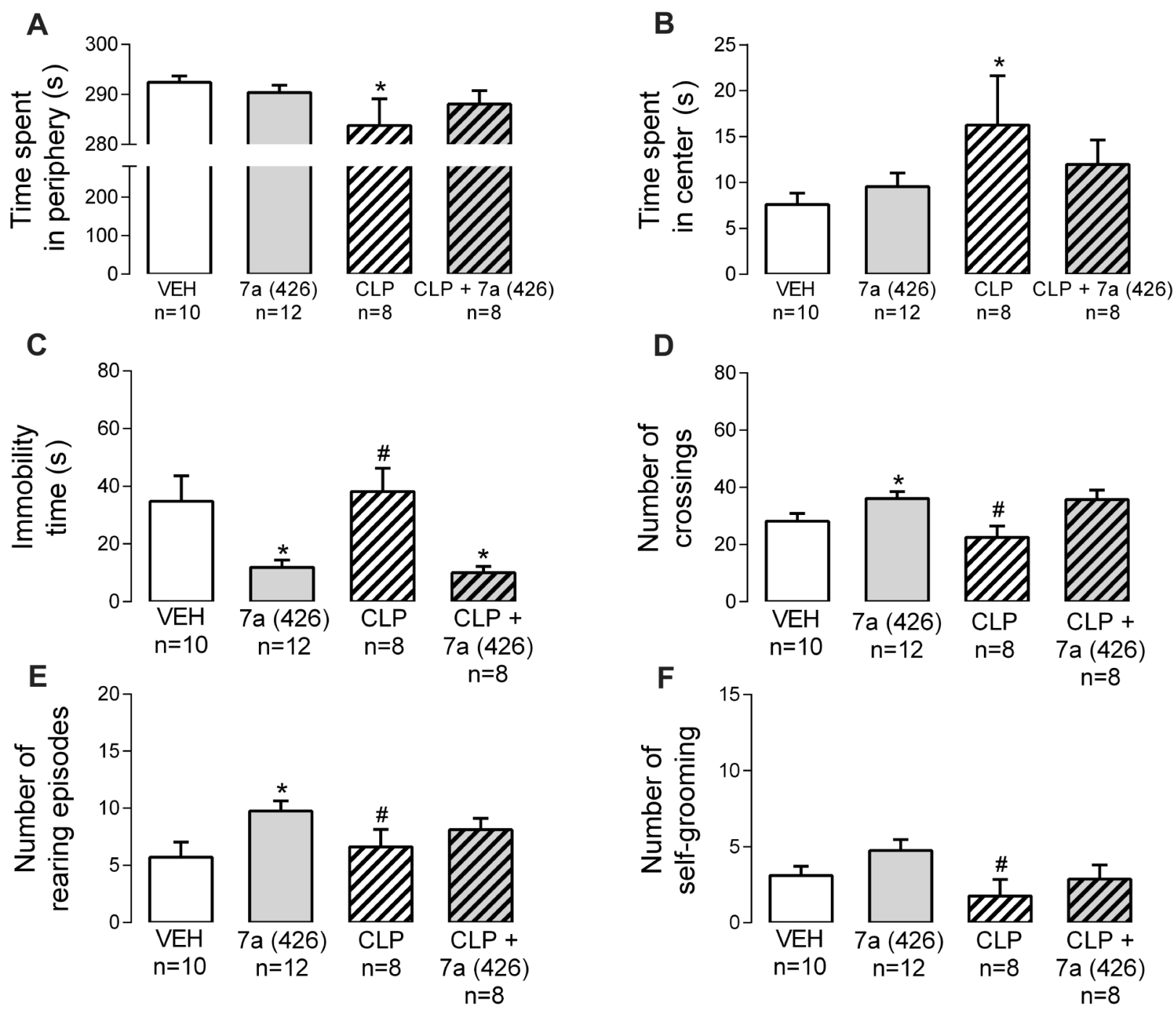

Figure 8. Contribution of dopaminergic receptors (as revealed by the antagonism with chlorpromazine) to the effects evoked by Bj-PRO$7 \mathrm{a}$ on locomotion/exploration in the open field. A. Time spent in periphery; $\mathbf{B}$, Time spent in center; $\mathbf{C}$, Immobility time; $\mathbf{D}$, Number of crossings; E, Number of rearing episodes; F, Number of self-grooming episodes. Experimental groups: VEH: vehicle $(0.9 \% \mathrm{NaCl}) ; 7 \mathrm{a}$ (426): Bj-PRO-7a (426 nmol/kg); CLP: chlorpromazine (2 mg/kg); CLP + 7a (426): co-administration of CLP and Bj-PRO-7a (426 nmol/ $\mathrm{kg}$ ). Data are reported as means $\pm \mathrm{SE}$. ${ }^{*} \mathrm{P}<0.05$ vs vehicle; ${ }^{\#} \mathrm{P}<0.05$ vs $7 \mathrm{a}$ (426) (one-way analysis of variance followed by Fisher's post hoc test).

In the case of the peptides from $B j$ venom, studies demonstrated that a peptide that is structurally similar to $B j-P R O-7 a$, the Bj-PRO-10c, was detected in mice brain following its peripheral injection. They observed that $B j$ PRO-10c was found at 5 and $60 \mathrm{~min}$ following its intraperitoneal injection (7). The route of injection and the timeframe of our experiments were similar to those employed in that study. About $2 \%$ of $B j-P R O-10 c$ was found in brain, which allowed drawing some hypotheses that should be considered: i) this $2 \%$ would be enough to produce the effects we found; ii) $B j-P R O-7 a$ is smaller compared to $B j-P R O-10 c$, and it may increase the possibilities of crossing the blood-brain barrier; iii) the peptide is able to cross the blood-brain barrier because of four benzene rings in its primary structure, which is strongly correlated with a degree of liposolubility (see Supplementary Figure S1); iv) $\mathrm{M}_{1} \mathrm{R}$ are broadly distributed in brain areas well known for controlling behavior, such as prefrontal cortex, amygdala, hippocampus, and striatum $(15,16)$, and the reversion of the Bj-PRO-7a-evoked anxiolysis by PZP is further evidence of a central effect for the heptapeptide. Therefore, we believe that Bj-PRO$7 a$ can change brain function and these changes partially depend on central $M_{1} R$. 
A

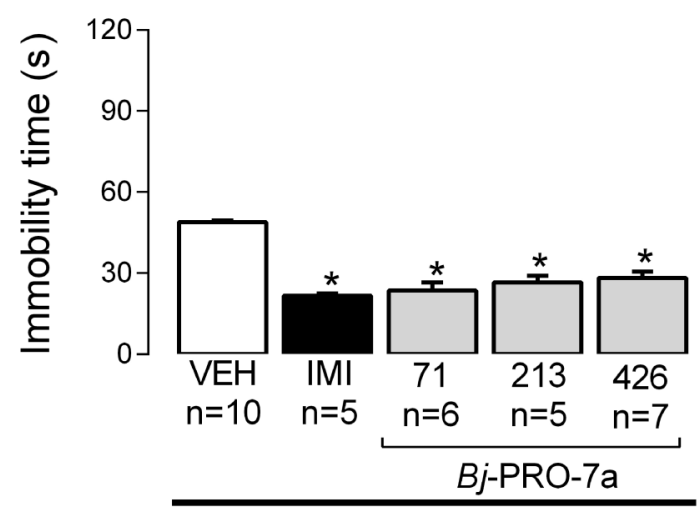

B

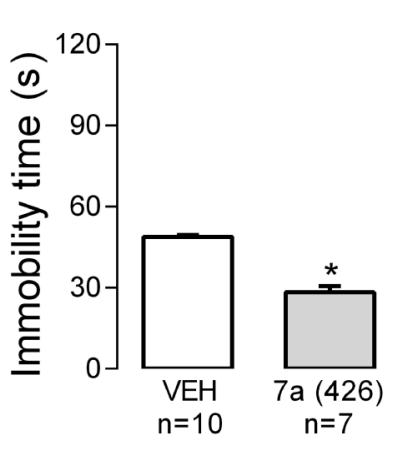

WT
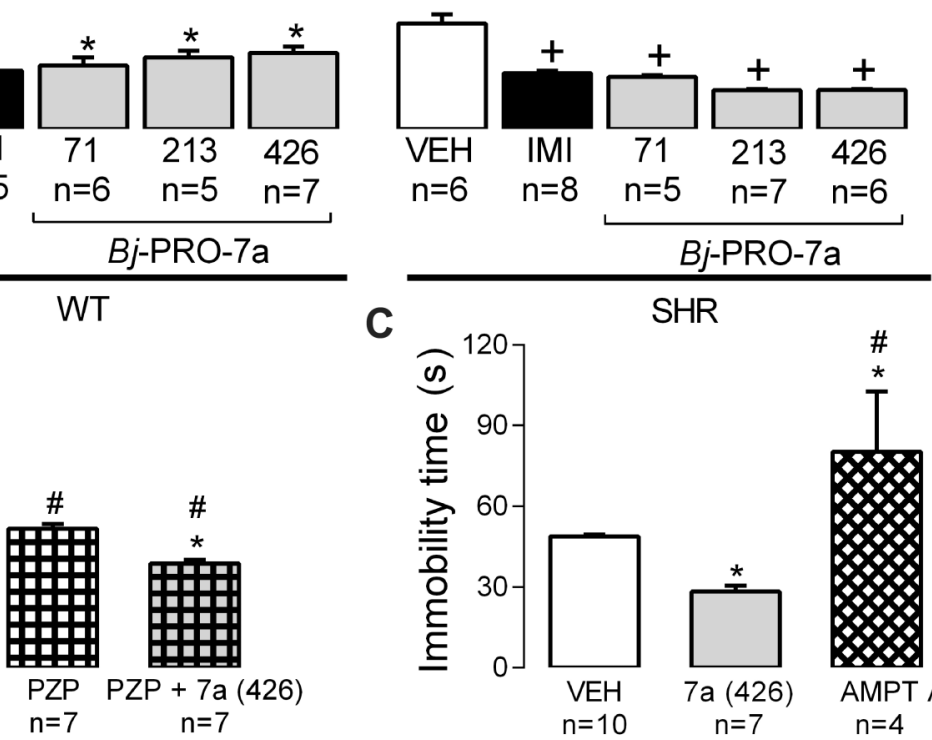

C
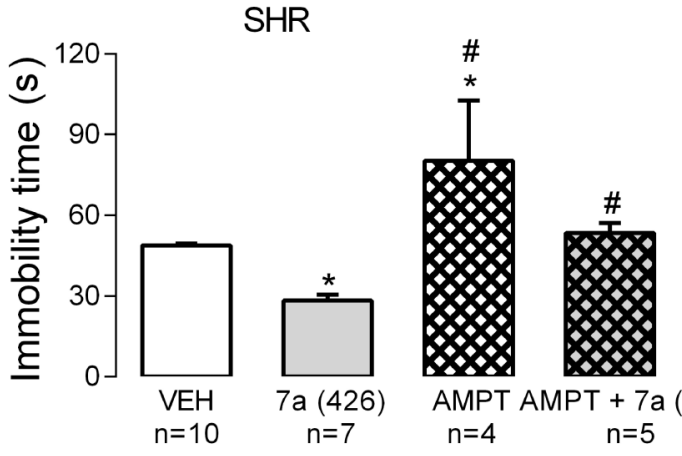

D

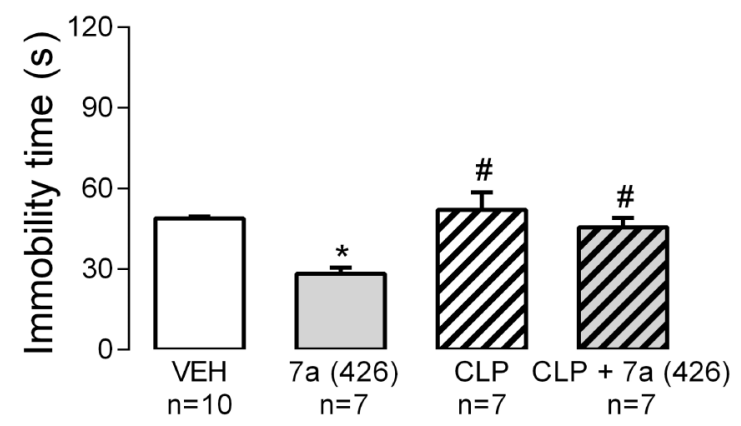

Figure 9. Antidepressant-like effects measured by the immobility time in the forced swimming test. A, Effects of Bj-PRO-7a on antidepressant-like behaviors in Wistar (WT) and spontaneously hypertensive rats (SHR). B, Contribution of muscarinic receptor subtype 1 (as revealed by the antagonism with pirenzepine - PZP) to the anti-depressant-like effects evoked Bj-PRO-7a. C, Contribution of catecholaminergic paths (as revealed by the depleter $\alpha$-methyl-DL-tyrosine: AMPT) to the effects evoked by Bj-PRO-7a. D, Contribution of dopaminergic receptors (as revealed by the antagonism with chlorpromazine - CLP) to the effects evoked by Bj-PRO-7a. Experimental groups: VEH: vehicle $(0.9 \% \mathrm{NaCl})$; IMI: imipramine $(15 \mathrm{mg} / \mathrm{kg}) ; 7 \mathrm{a}: \mathrm{Bj}-\mathrm{PRO}-7 \mathrm{a}(71,213$, or $426 \mathrm{nmol} / \mathrm{kg})$; PZP: pirenzepine $(852 \mathrm{nmol} / \mathrm{kg}) ; \mathrm{PZP}+7 \mathrm{a}(426)$ : co-administration of PZP and Bj-PRO-7a $(426 \mathrm{nmol} / \mathrm{kg}) ;$ AMPT $(200 \mathrm{mg} / \mathrm{kg})$; AMPT $+7 \mathrm{a}(426)$ : $\mathrm{co}-$ administration of AMPT and Bj-PRO-7a (426 nmol/kg); CLP: CLP (2 mg/kg); CLP+7a (426): co-administration of CLP and Bj-PRO-7a $(426 \mathrm{nmol} / \mathrm{kg})$. Data are reported as means \pm SE. ${ }^{*} \mathrm{P}<0.05$ vs VEH WT; ${ }^{+} \mathrm{P}<0.05$ vs VEH SHR; ${ }^{\#} \mathrm{P}<0.05$ vs $7 \mathrm{a}$ (426) [two-way analysis of variance followed by Bonferroni's test (panel A) and one-way analysis of variance followed by Fisher's post hoc test (panels B-D)].

Seminal studies already proposed the cholinergic system as one of the targets for snake toxins (14). Cholinergic neurons are broadly distributed in the CNS and modulate behavior and the cardiovascular and neuroendocrine systems (17). The central cholinergic system has been implicated in the physiopathology of affective disorders, mood regulation, sleep, and autonomic responses to stress (18). Besides, the central cholinergic system is able to affect - directly or indirectly - the function of other systems, such as the catecholaminergic. Dilsaver and Greden (1984) described that the activity of the tyrosine hydroxylases, the enzymatic limiting step for neuronal production of dopamine, adrenaline, and noradrenaline, is modulated by cholinergic mechanisms (19). 
In fact, the stimulation of cholinergic muscarinic receptors (mAChRs) reduces noradrenaline (NE) release in the hypothalamus (20), a highly permeable diencephalic region that may be considered a behavioral-autonomic interface. Additionally, mAChRs activation induces production and release of neurotrophic factors in neurons (21), which are important to reach antidepressant effects by producing synaptic plasticity, axon outgrowth, and neurogenesis. Therefore, the aforementioned reports are robust enough to justify the search for molecules that modulate cholinergic and catecholaminergic systems, since these paths are extremely important to the neurobiological organization of different functions and behaviors.

The function of central mAChRs varies according with the neural substrate in which they are expressed and with the subtype of these receptors. The mAChRs subtypes 1,3 , and 5 are attached to $\mathrm{Gq}$ protein with excitatory function. Conversely, the mAChRs subtypes 2 and 4 are attached to Gi protein, with inhibitory function (22). Gerber and colleagues showed the functional importance of $M_{1} R$ to the dopaminergic neurotransmission and to the control of locomotion. They found a positive correlation between the increases in $M_{1} R$ expression and release of dopamine in striatum (23). Much of this evidence was obtained using behavioral paradigms, which supports the $M_{1} R$ involvement in anxiety, depression, and in other neuronal disorders affecting behavior. A previous in vitro study (8) associated the cardiovascular effects of Bj-PRO-7a to $M_{1} R$. In light of these mechanistic clues, we tested the hypothesis that the anxiolytic and antidepressant-like effects of $B j-P R O-7 a$ would rely on $M_{1} R$. We found that behavioral effects evoked by Bj-PRO-7a were either reverted or attenuated by the simultaneous administration with PZP, which confirmed that the Bj-PRO-7a activity on behavior relies on $M_{1} R$. However, the reductions in the immobility time evoked by Bj-PRO-7a in the OF were unaffected by the concomitant antagonism of $M_{1} R$ with $\mathrm{PZP}$, which demonstrates the involvement of other pathways and synapses in the effects of Bj-PRO-7a on locomotion/exploration. Therefore, the subsequent question to be answered was about the other targets through which Bj-PRO-7a produces behavioral effects.

In order to uncover whether catecholaminergic pathways would be involved in the effects evoked by Bj-PRO$7 \mathrm{a}$, the methodological choice was to inhibit the tyrosine hydroxylase enzyme by AMPT. As a consequence, substantial depletions in dopamine, noradrenaline, and adrenaline levels, - as the natural sequence of reactions in the catecholaminergic metabolic paths - are found following injection of this enzymatic inhibitor (24). Our results showed that catecholaminergic pathways were substantially involved in the Bj-PRO-7a effects on anxiety and depression-like behaviors. The overactivation of the noradrenergic system can promote anxiety, especially due to malfunctioning of limbic structures (25). The locus coeruleus is the major brain source of noradrenergic neurons in charge of responding to stressors. Cholinergic neurons of the reticular system and gigantocellular tegmental area interact with locus coeruleus, modulating NE release (17). The projections from amygdala to locus coeruleus (26) is another possible pathway underlying interaction between central cholinergic and noradrenergic systems. Additionally, $M_{1} R$ s are expressed in basolateral amygdala, an important area involved in the sensorial circuitries (27). Therefore, the cholinergic system can regulate the activity of the noradrenergic system and consequently the organization of defense reactions. Molecules that are capable of attenuating increases in the firing rate of noradrenergic neurons in locus coeruleus are effective in reducing anxiety levels (28). In regard to our results, it is worth considering that the heptapeptide may regulate the NE release with consequent decreases in responsiveness to stress, thus allowing the rat to explore aversive areas. Notwithstanding the possible involvement of $M_{1} R s$ in anxiety, our results indicated that the activity of these receptors did not appear to contribute directly to the attenuation of depressive behavior exerted by Bj-PRO-7a.

Since dopamine is the major player in motivational processes that are impaired in mental disorders (29), we decided to investigate the contribution of dopaminergic receptors to the effects evoked by the heptapeptide. Despite finding that the antagonism of dopaminergic receptors with CLP itself promoted anxiolytic-like effect, the anxiolytic and antidepressant-like effects of Bj-PRO$7 a$ were reverted by the concomitant injection with CLP. The hypotheses that can be built from these observations are: i) the functionality of $M_{1} R$ implicates directly in dopamine release (23); ii) dopaminergic activity contributes to attenuate anxiety - probably by dopaminergic receptor subtype 2 - and depression; iii) Bj-PRO-7a would increase dopamine transmission on mesolimbic and mesocortical systems related to anxiolysis, motivation, exploration, and antidepressant effects (30).

Systems other than the noradrenergic, dopaminergic, and cholinergic may be involved in Bj-PRO-7a effects on behavior. Glutamatergic, gabaergic, and oxidonitrergic pathways would be good candidates for further studies. These hypotheses become even more plausible when previous reports on Bj-PRO-7a effects are kept in mind: i) the modulation of calcium transients in neurons (31) and ii) the activation of oxidonitrergic intracellular pathways by $M_{1} R$ agonism (8). Central NO increases extracellular concentrations of glutamate and $\gamma$-aminobutyric acid (GABA) and regulates $A C h$ and serotonin release in some brain regions governing locomotion and cognition (32). Interestingly, several antidepressants are capable of modulating central oxidonitrergic activity, such as serotonin reuptake inhibitors (33). The possibility that oxidonitrergic paths are recruited by Bj-PRO-7a to reach the antidepressant and anxiolytic-like effects is supported by previous findings: $B j-\mathrm{PROs}$ are able to activate NO release (4). Therefore, it cannot be neglected that central 
$\mathrm{NO}$ and GABA may be involved in anxiolysis and antidepressant-like effects of Bj-PRO-7a. This, however, needs to be addressed in further studies.

Our study indicated that the activation of cholinergic muscarinic receptors subtype 1 is required to reach the anxiolytic-like effect evoked by Bj-PRO-7a. The anxiolyticand antidepressant-like effects of Bj-PRO-7a seem to depend on catecholaminergic pathways and on dopamine receptors. Interestingly, the $\mathrm{M}_{1}$ Rs activation contributes to enhance memory and cognitive processes by inducing long-term potentials in hippocampus (34). Therefore, BjPRO-7a appears to be a promising innovative prototype capable of simultaneously treating the most prevalent psychiatric diseases such as anxiety and depression, through alternative mechanistic pathways with less adverse effects, contrary to many commercially available drugs often known for causing side effects such as drowsiness, hypnotic effects, cognitive and motor deficits, mental confusion, tolerance, and dependence (35). In spite of the noticeable Bj-PRO-7a cardiovascular effects, it is important to emphasize that the heptapeptide was able to reduce the blood pressure of hypertensive rats, i.e., the heptapeptide does

\section{References}

1. WHO (World Health Organization). Mental Health Atlas. Geneva: World Health Organization, 2018. License: CC BY-NC-SA 3.0 IGO. ISBN 978-92-4-151401-9. <https:// www.who.int/mental_health/evidence/atlas/mental_health_ atlas_2017/en/>

2. Osipov AV, Utkin YN. Snake venom toxins targeted at the nervous system. In: Gopalakrishnakone P, Inagaki $\mathrm{H}$, Vogel CW, Mukherjee A, Rahmy T, editors. Snake Venoms Toxinology: Springer, Dordrecht; 2017. p 189-214.

3. Camargo AC, lanzer D, Guerreiro JR, Serrano SM. Bradykinin-potentiating peptides: beyond captopril. Toxicon 2012; 59: 516-523, doi: 10.1016/j.toxicon.2011.07.013.

4. Morais KLP, lanzer D, Miranda JRR, Melo RL, Guerreiro JR, Santos RA, et al. Proline rich-oligopeptides: Diverse mechanisms for antihypertensive action. Peptides. 2013; 48: 124133, doi: 10.1016/j.peptides.2013.07.016.

5. lanzer D, Konno K, Marques-Porto R, Vieira Portaro FC, Stocklin R, Camargo AC, et al. Identification of five new bradykinin potentiating peptides (BPPs) from Bothrops jararaca crude venom by using electrospray ionization tandem mass spectrometry after a two-step liquid chromatography. Peptides 2004; 25: 1085-1092, doi: 10.1016/ j.peptides.2004.04.006.

6. Ianzer D, Santos RA, Etelvino GM, Xavier $\mathrm{CH}$, de Almeida Santos J, Mendes EP, et al. Do the cardiovascular effects of angiotensin-converting enzyme (ACE) I involve ACE-independent mechanisms? New insights from proline-rich peptides of Bothrops jararaca. J Pharmacol Exp Ther 2007; 322: 795-805, doi: 10.1124/jpet.107.120873.

7. Silva CA, Portaro FC, Fernandes BL, lanzer DA, Guerreiro JR, Gomes CL, et al. Tissue distribution in mice of BPP 10c, a potent prolinerich anti-hypertensive peptide of Bothrops not promote hypotension in the normotensive strain (6). Altogether, these central and peripheral effects indicate $B j$ PRO-7a to be a good prototype candidate for treating disorders involving cholinergic and catecholaminergic targets.

\section{Supplementary material}

Click here to view [pdf].

\section{Acknowledgments}

This work was supported by DOCFix CAPES/FAPEG 2014, Central de Aperfeiçoamento de Pessoal de Nível Superior (CAPES), Fundação de Amparo à Pesquisa do Estado de Goiás (FAPEG, INCT NanoBioFar), and Conselho Nacional de Desenvolvimento Científico e Tecnológico (CNPq-Universal 406393/2018-4; PQ 308156/20188). L.C. Turones, G. Camargo-Silva, and K.R. da Cruz were recipients of CAPES Master Fellowships at the Graduate Program in Biological Sciences-UFG. L.L. Reis-Silva was the recipient of CNPq honors fellowship (PIBIC). D. lanzer was supported by DCR CNPq/FAPEG. jararaca. Toxicon 2008; 51: 515-523, doi: 10.1016/j.toxicon. 2007.11.003.

8. Negraes PD, Lameu C, Hayashi MA, Melo LM, Camargo $\mathrm{AC}$, Ulrich $\mathrm{H}$. The snake venom peptide Bj-Pro-7a Is a M1 muscarinic acetylcholine receptor agonist. Cytometry $A$ 2011; 79: 77-83, doi: 10.1002/cyto.a.20963.

9. Morilak DA, Frazer A. Antidepressants and brain monoaminergic systems: a dimensional approach to understanding their behavioural effects in depression and anxiety disorders. Int J Neuropsychopharmacol 2004; 7: 193-218, doi: $10.1017 / \mathrm{S} 1461145704004080$.

10. da Cruz KR, Turones LC, Camargo-Silva G, Gomes KP, Mendonça MM, Galdino P, et al. The hemoglobin derived peptide LVV-hemorphin-7 evokes behavioral effects mediated by oxytocin receptors. Neuropeptides 2017; 66: 59-68, doi: 10.1016/j.npep.2017.09.002.

11. Witkin JM, Overshiner C, Li X, Catlow JT, Wishart GN, Schober DA, et al. M1 and M2 muscarinic receptor subtypes regulate antidepressant-like effects of the rapidly acting antidepressant scopolamine. J Pharmacol Exp Ther 2014; 351: 448-456, doi: 10.1124/jpet.114.216804.

12. Degroot A, Nomikos GG. Fluoxetine disrupts the integration of anxiety and aversive memories. Neuropsychopharmacol 2005; 30: 391-400, doi: 10.1038/sj.npp.1300624.

13. Terzioglu B, Kaleli M, Ayd̆in B, Ketenci S, Cabadak H, Goren $M Z$. Increased noradrenaline levels in the rostral pons can be reversed by M1 Antagonist in a rat model of posttraumatic stress disorder. Neurochem Res 2013; 38: 17261733, doi: 10.1007/s11064-013-1076-2.

14. Chang CC, Lee CY. Isolation of neurotoxins from the venom of bungarus multicinctus and their modes of neuromuscular blocking action. Arch Int Pharmacodyn Ther 1963; 144: 241-257. 
15. Flynn DD, Ferrari-DiLeo G, Mash DC, Levey Al. Differential regulation of molecular subtypes of muscarinic receptors in Alzheimer's disease. J Neurochem 1995; 64 1888-11891, doi: 10.1046/j.1471-4159.1995.64041888.x.

16. Levey AI, Edmunds SM, Koliatsos V, Wiley RG, Heilman CJ. Expression of m1-m4 muscarinic acetylcholine receptor proteins in rat hippocampus and regulation by cholinergic innervation. J Neuroscience 1995; 5: 4077-4092, doi: 10.1523/JNEUROSCI.15-05-04077.1995.

17. Dilsaver SC. Cholinergic mechanisms in affective disorders. Acta Psychiatr Scand 1986; 74: 312-334, doi: 10.1111/ j.1600-0447.1986.tb06250.x.

18. Dilsaver SC. Cholinergic mechanisms in depression. Brain Res 1986 396: 285-316, doi: 10.1016/0165-0173(86)90016-0.

19. Dilsaver SC, Greden JF. Antidepressant withdrawalinduced activation (hypomania and mania): mechanism and theoretical significance. Brain Res 1984; 319: 29-48, doi: 10.1016/ 0165-0173(84)90028-6.

20. Westfall TC. Effect of acetylcholine on the release of $\left({ }^{3} \mathrm{H}\right)$ norepinephrine by nicotine and potassium chloride from rat brain slices. In: Usdin E, Snyder SH, editors. Frontiers of catecholamine research. New York: Pergamon Press; 1973. p 617-618, doi: 10.1016/B978-0-08-017922-3.50113-1.

21. Navakkode S, Korte M. Cooperation between cholinergic and glutamatergic receptors are essential to induce BDNFdependent long-lasting memory storage. Hippocampus 2012; 22: 335-346, doi: 10.1002/hipo.20902.

22. Eglen RM. Overview of Muscarinic Receptor Subtypes. Handb Exp Pharmacol 2012: 3-28, doi: 10.1007/978-3-64223274-9

23. Gerber DJ, Sotnikova TD, Gainetdinov RR, Huang SY, Caron MG, Tonegawa S. Hyperactivity, elevated dopaminergic transmission and response to amphetamine in M1 muscarinic acetylcholine receptor-deficient mice. Proc Natl Acad Sci USA 2001; 98: 15312-15317, doi: 10.1073/pnas. 261583798.

24. Corrodi H, Hanson LC. Central effects of an inhibitor of tyrosine hydroxylation. Psychopharmacologia 1966; 10 : 116-125, doi: 10.1007/BF00455973.

25. Lange $\mathrm{C}$, Irle $\mathrm{E}$. Enlarged amygdala volume and reduced hippocampal volume in young women with major depression. Psychol Med 2004; 34: 1059-1064, doi: 10.1017/S0033291703001806.

26. Jacobs BL, Abercrombie ED, Fornal CA, Levine ES, Morilak DA, Stafford IL. Single-unit and physiological analyses of brain norepinephrine function in behaving animals. Prog Brain Res 1991; 88: 159-165, doi: 10.1016/S0079-6123(08) 63805-4.

27. LeDoux JE. Emotion circuits in the brain. Annu Rev Neurosci 2000; 23: 155-184, doi: 10.1146/annurev.neuro. 23.1.155.

28. Bremner JD, Krystal JH, Southwick SM, Charney DS. Noradrenergic mechanisms in stress and anxiety: I. Preclinical studies. Synapse 1996; 23: 28-38, doi: 10.1002/(SICI) 1098-2396(199605)23:1 < 28::AID-SYN4 > 3.0.CO;2-J.

29. Pignatelli $M$, Bonci $A$. Role of dopamine neurons in reward and aversion: a synaptic plasticity perspective. Neuron 2015; 86: 1145-1157, doi: 10.1016/j.neuron.2015.04.015.

30. Rezayof A, Hosseini SS, Zarrindast MR. Effects of morphine on rat behaviour in the elevated plus maze: the role of central amygdala dopamine receptors. Behav Brain Res 2009; 202: 171-178, doi: 10.1016/j.bbr.2009.03.030.

31. Lameu C, Hayashi MA, Guerreiro JR, Oliveira EF, Lebrun I, Pontieri V. The central nervous system as target for antihypertensive actions of a proline-rich peptide from Bothrops jararaca venom. Cytometry $A$ 2010; 77: 220-230, doi: 10.1002/cyto.a.20860.

32. Segovia G, Porras A, Mora F. Effects of a nitric oxide donor on glutamate and GABA release in striatum and hippocampus of the conscious rat. Neuroreport 1994; 5: 1937-1940, doi: 10.1097/00001756-199410000-00024.

33. Dhir A, Kulkarni SK. Nitric oxide and major depression. Nitric Oxide 2011; 24: 125-131, doi: 10.1016/j.niox.2011.02.002.

34. Dennis SH, Pasqui F, Colvin EM, Sanger H, Mogg AJ, Felder CC, et al. Activation of muscarinic M1 acetylcholine receptors induces long-term potentiation in the hippocampus. Cereb Cortex 2016; 26: 414-426, doi: 10.1093/cercor/ bhv227.

35. Ashton AK, Jamerson BD, L Weinstein W, Wagoner C. Antidepressant-related adverse effects impacting treatment compliance: results of a patient survey. Curr Ther Res Clin Exp 2005; 66: 96-106, doi: 10.1016/j.curtheres.2005.04.006. 